\title{
How Can You Treat Rheumatoid Arthritis without Using Corticosteroids and Immunosuppressive Medications?
}

\author{
Huang, W. L
}

Infectious Diseases, General Practice, Nutrition, Acupuncture and Pain Management Specialist. Medical Acupuncture and Pain Management Clinic, Franca, São Paulo, Brazil.

*Corresponding Author: Huang Wei Ling, MD, Rua Homero Pacheco Alves, 1929, Franca, São Paulo, 14400-010, Brazil.

\section{Abstract}

Statement of the Problem: Rheumatoid arthritis (RA) is a chronic autoimmune inflammatory disease, which affects approximately $1 \%$ of the world's adult population. It is characterized by the inflammation of synovial tissue from multiple articulations. According to traditional Chinese medicine (TCM) theory, RA is categorized under the "Bi" or impediment disease, a group of diseases caused by the invasion of Wind, Cold, Dampness, or Heat pathogen on the meridians involving muscles, sinews, bones, and joints.

Purpose: To demonstrate that patient with rheumatoid arthritis can be treated without the use of corticosteroids and immunosuppressive medications.

Methods: One case report of a 26-year-old male, with rheumatoid arthritis since 2015, and was using immunosuppressive medications and corticosteroids to control the pains in all joints. He began to treat his pains with a Chinese medicine doctor who advise him to avoid all dairy products and other foods that could imbalance the internal energy. He also was submitted to auricular acupuncture, apex ear bloodletting and radiesthesia procedure. All his seven chakras' energies centers were in the lowest level of energy (rated 1 out of 8) and he was medicated with homeopathies according to the theory Constitutional Homeopathy of the Five Elements based on Traditional Chinese Medicine and crystal-based medications for a period of one year or more.

Results: The patient improved from his symptoms after the oriental medicine tools and avoiding all dairy products and after less one month of treatment he was able to withdrawn corticosteroids and immune suppressant medications.

Conclusion: rheumatoid arthritis patients can be treated without using corticosteroids or immunosuppressive medications. To this end, the physician need to understand the factors involving the formation of disease in the energy point of view. Protections against the invasion of external pathogenic factors, orientations regarding the type of foods recommended to eat and replenishment the chakras' energies centers with homeopathies and crystal-based medications are important to fortify the immune system of the patient and rebalance all the internal energies of the patient.

Keywords: Rheumatoid arthritis, Chinese dietary counseling, Energy, Traditional Chinese Medicine, Chakras, Homeopathy, Hippocrates.

\section{INTRODUCTION}

According to Western medicine, rheumatoid arthritis (RA) is a chronic autoimmune inflammatory disease, which affects approximately $1 \%$ of the world's adult population. It is characterized by the inflammation of synovial tissue from multiple articulations, leading to tissue destruction, pain, deformities and reduced quality of life. Rheumatoid arthritis etiology is complex and largely unknown, although studies support the influence of genetic and environmental factors on its pathogenesis. [1]

In this article, the author will show a different 
How Can You Treat Rheumatoid Arthritis without Using Corticosteroids and Immunosuppressive Medications?

view of rheumatoid arthritis, using the teachings of Hippocrates who said "foolish the doctor who despises the knowledge acquired by the ancients." So, the author will use traditional Chinese medicine reasoning to explain all the concepts involved in the formation of the disease, in the energy point of view and how to treat patients with rheumatoid arthritis without using corticosteroids and immunosuppressive medications[2]

\section{PURPOSE}

The purpose of this study is to demonstrate that patient with rheumatoid arthritis can be treated without the use of corticosteroids and immunosuppressive medications.

\section{METHODS}

Through an extensive literature review using PubMed and NCBI indexed articles related on rheumatoid arthritis according to Western medicineand traditional Chinese medicine point of view. Also, the author used one case report to illustrate this article.

\section{CASE REPORT}

This patient was a 26-year-old male the author treated him in 2018, with diagnosis of rheumatoid arthritis since 2013. He was using immunosuppressive medications and corticosteroids to control the pains in all joints, without any improvement of his pains and

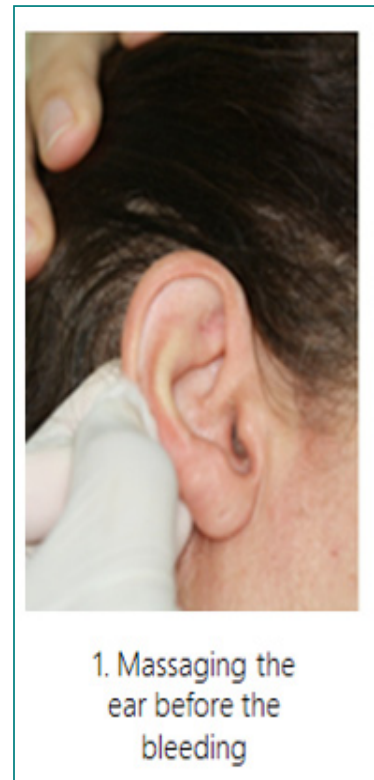

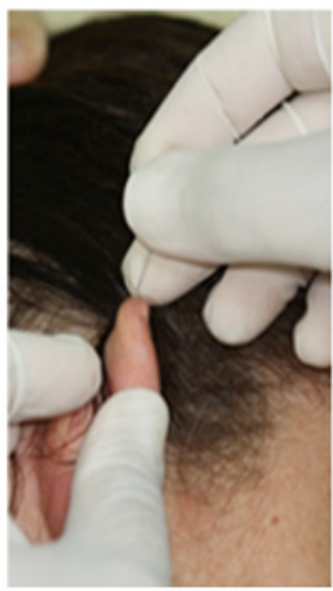

2. The ear is bent and the needle is inserted on the top of it

he told her that he was very tired to need to use such medications to control his pain and disease.

His diagnoses in TCM were deficiency of Yin (feel Hot), Yang (feel cold feet), Blood (do not daily bowel movement), Qi (sweets during the day) and Heat retention (dry mouth, bad breath, itchiness).

He began to treat his condition using Chinese dietary counseling consisted in avoiding all dairy products, cold water, raw food and sweets.

The second group of foods that was orientated to avoid was fried foods, chocolate, honey, melted cheese, coconut, alcoholic beverages.

The third group of foods to avoid was coffee, soda and matte tea.

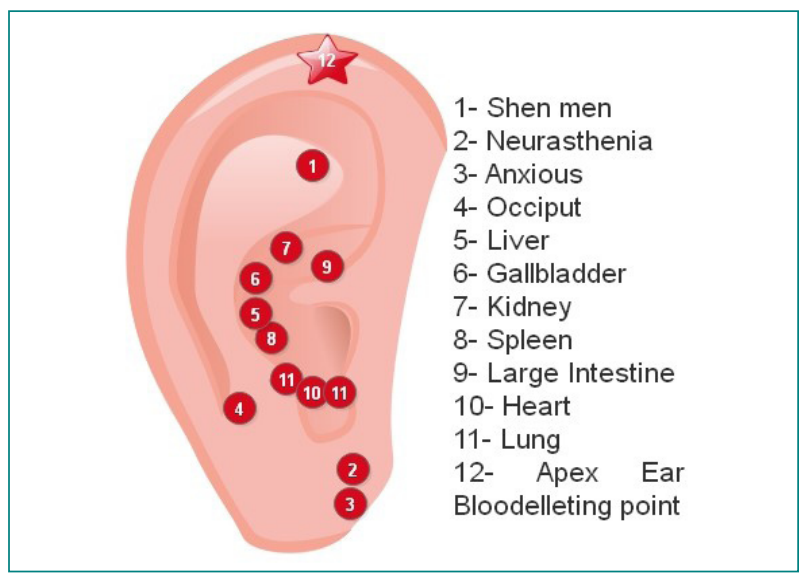

Figure 1. Auricular Acupuncture points used

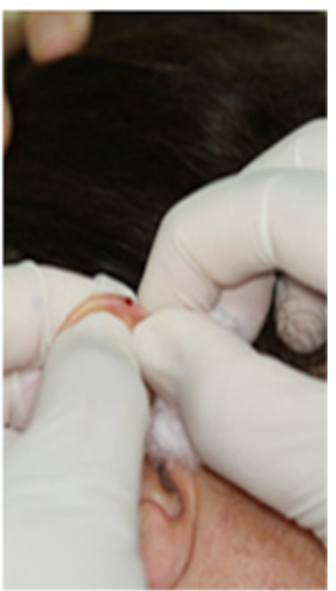

3. Five drops are squeezed from the top of the ear

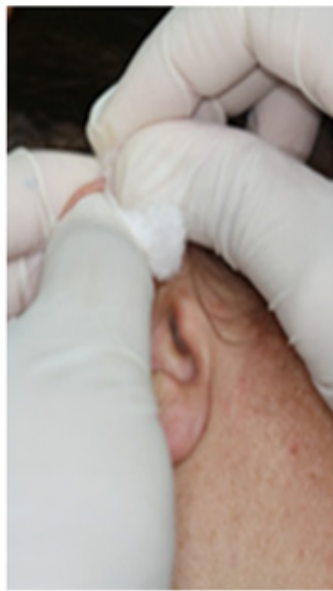

4. Pressure with fingers is done to increase bleeding and a cotton ball is used to clean it.

Figure 2. Apex ear bloodletting to take out the Heat 
How Can You Treat Rheumatoid Arthritis without Using Corticosteroids and Immunosuppressive Medications?

The patient also was submitted to auricular acupuncture with apex ear bloodletting using mustard seeds twice a week with the intention to balance his internal energies of Yin, Yang, Qi, Blood and take out Heat retention, as you can see in the Figures 1 and 2.

The patient was recommended to protect even the entire joint with light clothing; for example, avoid shorts that do not cover the knees, blouses with shorter sleeves, use a very light fabric to prevent the Wind from entering. He was also orientated to avoid the exposition to fan, air-conditioned, open the fridge, walk barefoot, stay with wet hair after shower. Also, it was orientated to take out the wet clothes after doing any exercises.

The author also submitted him to radiesthesia procedure to see his chakras energies level. All his seven chakras' energies centers were in the lowest level of energy (rated 1 out of 8).

He was medicated with high diluted medications (homeopathies and crystal-based medications) for a period of one year or more, according to the theory created by the author entitled Constitutional Homeopathy of Five Elements based on Traditional Chinese Medicine. The medications used were following the sequence: Sulphur 30CHXX-20 (single dose), Calcarea carbonica 30CHXX-20 (single dose), Silicea 30CHXX-20 (single dose), Natrum muriaticum 30CHXX-20 (single dose), Phosphorus 30CHXX-20. The homeopathies were prescribed to be taken in a single dose with a hiatus of three days between the medications, necessarily on the sequence described. Two months later, a new sequence of treatment was started, with the same medications taken on the same order, but now with 200CHXX-20 instead of 30 CHXX20. Two months later, a new sequence of treatment was started, as the others, with the same medications taken on the same order, now with 1000CHXX-20 instead of 200CHXX-20. Two months later, a new sequence of treatment was started, as the others, with the same medications taken on the same order, now with 10000CHXX-20 instead of 1000CHXX-20. Two months later, a new sequence of treatment was started, as the others, with the same medications taken on the same order, now with 50000CHXX-20 instead of 10000CHXX-20.

\section{RESULTS}

The patient improved from his symptoms after the Chinese medicine tools (Chinese dietary counseling, auricular acupuncture with apex ear bloodletting) and replenishment of the chakras' energies centers with homeopathies using Constitutional Homeopathy of the Five Elements Based on Traditional Chinese Medicine and crystal-based medications and after less than one month of treatment he was able to withdrawn his high concentrated medications such as corticosteroids and immune suppressant medications and never need to use them anymore.

\section{Discussion}

The author used to construct this article, thoughts of Hippocrates that said that it is important to consider other ancient medical traditions prior to the knowledge we have nowadays. Another quote from him used by the author was make you food your medicine and your medicine your food. The third quote from him used by the author is that it is more important to know which person has a disease than to know which disease a person has. [2]

To explain how was the reasoning used by the author to treat this patient in question and all of her patients, she would like to show the history of one specific patient that she treated in 2006 and became the cornerstone of all her treatments. [3-9]

The patient in question was a 70-year-old male patient who searched for the author's clinic because he was treating his leg pain with anti-inflammatory medications for 6 months with no improvement. He searched for the treatment using acupuncture and that's why he went to the author's clinic. [3-9]

According to TCM he was diagnosed with Kidney-Yang deficiency (feels cold in his extremeties). [3-9]

He started the treatment with Chinese dietary counseling, being recommended to avoid dairy products, raw foods, cold drinks, sweets. [3-9]

Another group of foods that was recommended to avoid was chocolate, honey, eggs, fried foods, coconut, melted cheese and alcoholic beverages. [3-9]

And the last group was to avoid coffee, soda and mate tea. [3-9]

It was also made systemic acupuncture and auricular acupuncture with apex ear bloodletting twice a week. [3-9] 
How Can You Treat Rheumatoid Arthritis without Using Corticosteroids and Immunosuppressive Medications?

With the treatment done, the painin thelegs diminished and the patient was submitted to an interview 30 days after the treatment. In this interview, the patient revealed his eye pressure had also lowered, as his ophthalmologist confirmed. During the treatment, he had not reported to be treating glaucoma in the last 40 years with only little improvement of his condition. [3-9]

This unusual case became the cornerstone of all the author's studies in the field, trying to comprehend how the treatment focused on the root of the problem could treat different diseases and symptoms simultaneously and using the same methods. [3-9]

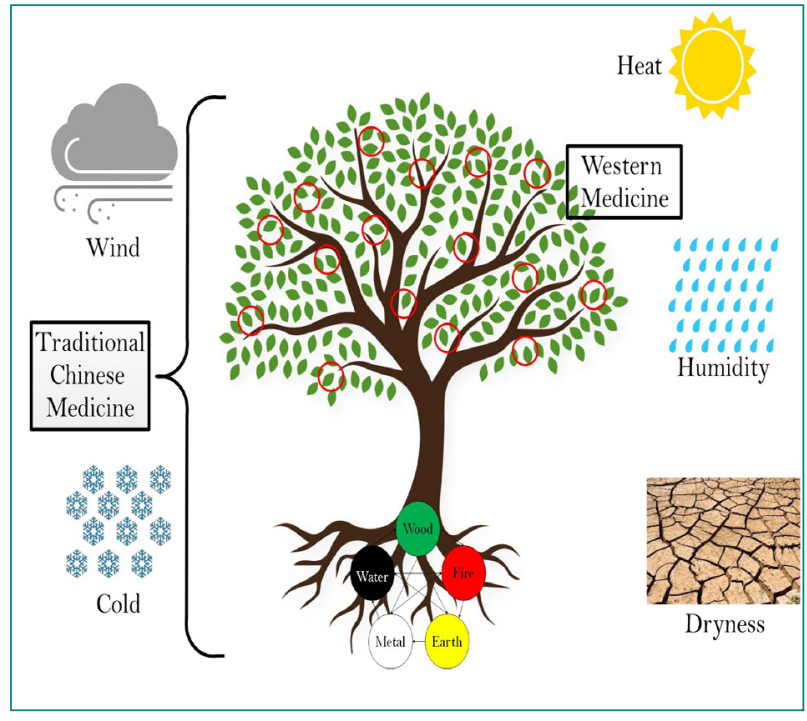

Figure 3. Metaphor of the tree showing differences in the view point of Western medicine and traditional Chinese medicine.

To show you how the author constructs the reasoning to treat this patient with rheumatoid arthritis, she will explain the differences between Western medicine and TCM point of view, using a metaphor of the tree (Figure 3). [3-9]

This tree has a trunk with several branches and each branch represents one medical specialty. Coming out of each branch there are many leaves that represent the symptoms and diseases treated by each medical specialty. So the focus of Western medicine is only the visible part of the tree, the branches and leaves. In this case, rheumatoid arthritis is treated in the leaf level (Figure 3). The intention of this article is to address the energy imbalances that are in the root level, invisible by the naked eye, corresponding to imbalances of the
Yin and Yang energy and the disequilibrium in the Five Elements theory that is leading to the symptoms in the leaf level. As you can see in this Figure 3, the tree is surrounded by the external pathogenic factors that are Cold, Wind, Dryness, Humidity and Heat. The significance of these factors in the formation of rheumatoid arthritis symptoms is the major importance to understand to know how to do the treatment and improve the symptoms and give more quality of life of these patients. [3-9]

The treatment in the root level treating the imbalance between Yin, Yang, Qi and Blood was a theme that the author presented in 2015 at the Acupuncture Research Conference that took place at the Harvard University, in Boston that states that treating these energies imbalances, the physician will be able to treat all the patients' symptoms (physical or emotional) at the same time, even if the physician does not know that the patient has such symptom. [10]

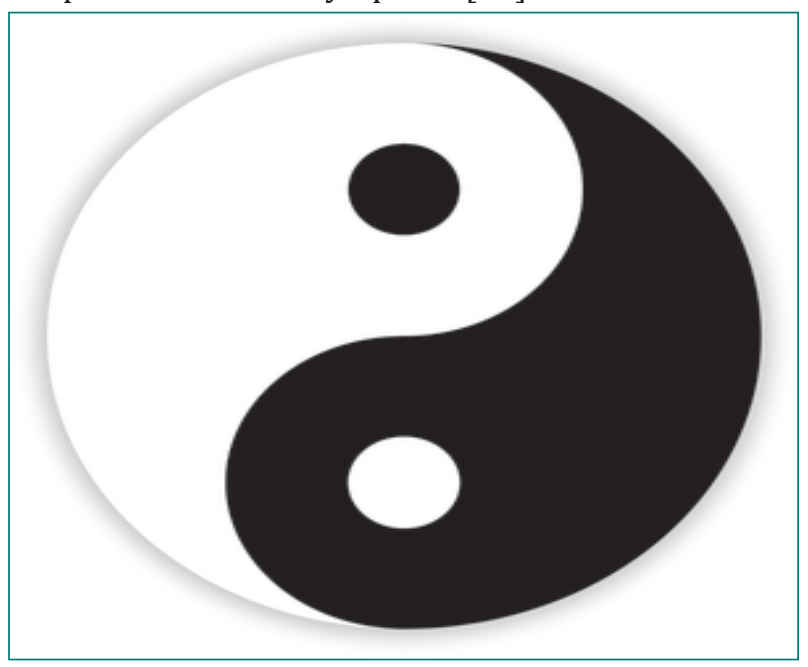

Figure 4. Yin and Yang symbol

According to TCM, all the treatments are based on the equilibrium between Yin and Yang energies (Figure 4). They are opposite forces, but complementary, such as day and night, men and women, good and bad, etc. [3-9]

The symmetrical arrangement of the dark Yin and the light Yang is not static, as we can see in the symbol in the Figure 4. [3-9]

The relationship between Yin and Yang energies is a rotational symmetry, and a continuous cyclic movement. The Yang cyclically returns to its beginning, the Yin reaches its apogee and yields its place to the 
How Can You Treat Rheumatoid Arthritis without Using Corticosteroids and Immunosuppressive Medications?

Yang. The two points in the diagram symbolize the idea that every time each force reaches its extreme point, it manifests within itself the seed of its opposite. [3-9]

If there are imbalances between these two energies, symptoms occur, and if not properly treated, they can result in diseases in the future. [3-9]

Inside the body, Yin is associated with organ tissue, while Yang is associated with organ function. With a Yin-deficiency, organs are deficient in nourishment, while a Yang-deficiency reflects a functional or performance deficiency in an organ or organ system. [3-9]

The disequilibrium between these two energies leads to the formation of symptoms and diseases. One example of Yin deficiency is women at menopause comprehended by symptoms of hot flashes, as you can see in the Figure 5, in columns wrapped in red, that the Yang force is bigger than the Yin force. Another example of Yang deficiency is when the patient feels cold mainly in the extremities, characterized in the Figure 5, in the columns wrapped in blue, when Yang energy is less than Yin. This deficiency (Yang deficiency) usually leads to retention of liquid in the body and the patient could complain of difficulty in loss weight, could have difficult in pregnancy, urinary incontinence, etc. [3-9]

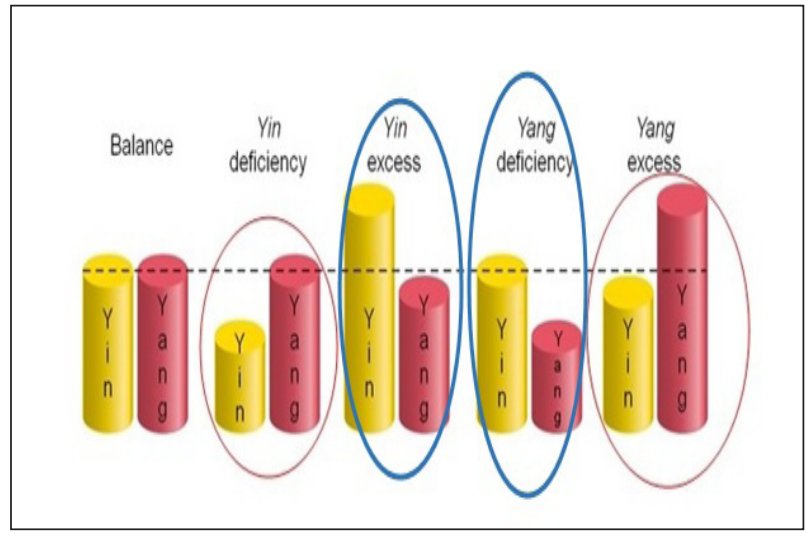

Figure 5. Yin and Yang in balance and imbalanced states.

According to traditional Chinese medicine, all autoimmune disease is characterized by Yin deficiency condition, shown in the Figure 5. [11]

For Yin and Yang energy to flow, two other energies have to work in harmony with it: $Q i$ and Blood. $Q i$ is basically the energy that circulates throughout your body. Qi moves the Blood, that means if Qi stays stagnant long enough, then the local body fluids that are supposed to be flowing begin to stagnate as well. To achieve health, the main aim is to balance these four energies Yin, Yang, Qi and Blood, shown in the Figure 6. [3-9]

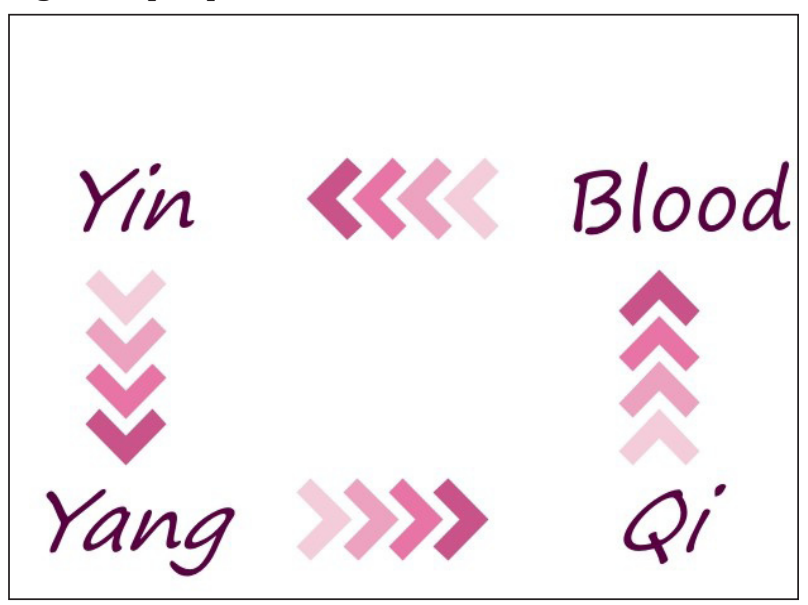

Figure 6. Yin, Yang, Qi and Blood relationship

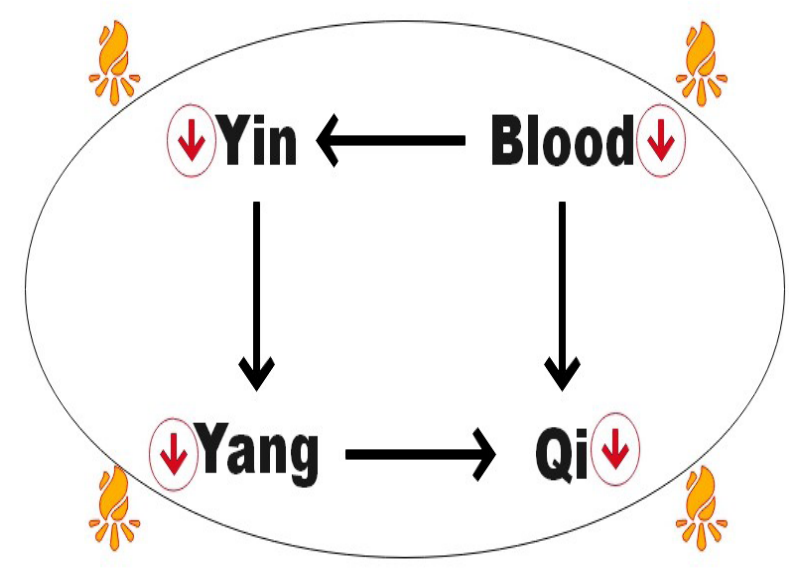

Figure 7. Formation of Heat when there are energies deficiencies between Yin or Yang or Qi or Blood or a combination of these 4 forces deficiencies.

The lack of energy of one of these energies or a combination of them can lead to the formation of internal Heat inside the body, as you can see in the Figure 7. [3-9]

As said by Hippocrates, make your food you medicine, and your medicine your food. [2]

The first step in all kinds of treatments made by the author is to change the wrong diet habits of her patients that is causing energies imbalances of the internal energy. The Chinese nutritional therapy is 
How Can You Treat Rheumatoid Arthritis without Using Corticosteroids and Immunosuppressive Medications?

different from the Western dietary therapy because in the Chinese dietary therapy, they study the energy point of view of each food that in Western medicine, they study only the components of these foods regarding the percentage of proteins, carbohydrates, lipids, vitamins, etc... [12-13]

The patient described in the case report had all the four energies deficiencies (Yin, Yang, Qi and Blood).[3-9]

The foods that the patient needs to avoid to prevent the formation of Yin and Yang deficiency are the ingestion of coffee, soda, mate tea. [3-9]

These kinds of foods are very common in Brazil specially the ingestion of coffee by all the populations since childhood or since they are in the mother's belly. This energies imbalances usually are caused by chronic ingestion of such foods during years and the treatment, when you take out or reduce these foods from the patients, they usually want to be cured from this condition in the same week, but the author usually orientate the patient that as they induced this disequilibrium during their whole life, the recovery of this energy is gradually and not instantaneous. [3-9]

Although TCM does not refer to rheumatoid arthritis by name of the disease, it does address diseases called Bi syndromes. Bi means obstruction and it is caused by the invasion of external pathogenic factor pathogens creating blockage in the energy meridians channels. This blockage prevents $Q i$ and Blood from travelling through the channels smoothly and performing their functions of warming and nourishing the joints and extremities, thus causing serious symptoms. [13]

TCM says: 'The four external evils known as Wind, Cold, Heat and Dampness attack the human body and cause Bi syndromes.' TCM teaches that exterior pathogens can attack the body when $Q i$ and Blood are deficient and so cannot protect the body very well. This attack, coupled with the $Q i$ and Blood deficiency, can result in Bi syndrome. Thus, the etiology of Bi syndrome involves some combination of the external pathogens, such as Wind, Cold, Heat and Dampness that invade the body as a result of an internal deficiency of $Q i$ and Blood. [13]

In the case of the patient reported in this article, he had Qi and Blood deficient, facilitating the invasion of the external pathogenic factor, leading to Bi syndrome, characterized by the pain and stiffness in the joints he was complaining. $[13,14]$
In TCM, Wind is the most important pathogenic factor as it always leads to other pathogens to attack the body, such as Cold. This attack can occur if the weather changes suddenly, if people live in a Cold or rainy place or wear few clothes, when people jog in extremely Cold and Damp weather or if people sweat profusely thereby causing the pores to open. Any of these factors can facilitate the invasion of external Wind, Cold, Dampness or Heat, and that is why it was orientated to avoid Wind and Cold to prevent the aggravation of his pain condition.[14]

According to TCM, in the acute phase of the disease, the treatment aims to dispel pathogenic Heat and Cold, remove obstruction in the channels, and dispel pathogenic Wind and Dampness. In the chronic phase, the treatment needs to nourish the Liver and Kidney, remove Blood stasis and smooth the channels. [14]

These pathogens can also entrance in the body through the foods, as the main foods that have Cold energy are all the fruits and vegetables, with the exception of papaya, lemon and apple that are neutral in energy. In this case, it was orientated the patient to avoid raw food and fruits and it was necessary to cook or bake them before eating, to neutralize the Cold energy of these kinds of foods. [15]

In the Chinese medicine literature, these kinds of patients are more prompt to have rheumatoid arthritis symptoms due to deficiency of Blood and Qi. In the case report, the patient has not only Blood and $Q i$ deficiency, but also, Yin and Yang deficiency. To treat these four energies deficiencies, the first tool used by the author was to correct the wrong dietary habits of this patient, that was conducive to these energies imbalances, leading a weakened state of energy and favoring the invasion of the external pathogenic factor, aggravating the pain in the joints presented by this patient. [14]

To improve the Blood production, it was necessary to take out all dairy products because dairy products can induce the malabsorption of nutrients by Spleen and pancreas meridian and leading subsequently to Blood deficiency. The ingestion of dairy products is responsible for all joint pain symptoms, according to TCM, and in the daily practice of the author, every patient with pain in the joints, the first step is to take out all dairy products. This recommendation includes the dairy presented in the cakes, pudding, ice cream, chocolate, pizza, lasagna, etc. All the foods that contains dairy products should be avoided. [15] 
How Can You Treat Rheumatoid Arthritis without Using Corticosteroids and Immunosuppressive Medications?

The use of sweet foods also should be avoided, even if without any calories, because the sweet taste foods also lead to imbalance of Spleen-pancreas meridian, leading to less absorption of nutrients, leading to anemia or Blood deficiency. That is why it is necessary to advice the patient to avoid sweeteners in coffee, tea, soda because even without calories, could induce Spleen-pancreas deficiency, leading to Blood deficiency in the future because of the sweet taste. [15]

\begin{tabular}{|c|l|c|l|l|}
\hline \multicolumn{5}{|c|}{ Progression health to disease } \\
\hline Phase & \multicolumn{1}{|c|}{ Organ } & Exams & Energy Reserve & \multicolumn{1}{|c|}{ Symptom } \\
\hline 1 & $\begin{array}{l}\text { Slowing down of } \\
\text { organ functions }\end{array}$ & Normal & Normal & $\begin{array}{l}\text { Without critical } \\
\text { symptoms }\end{array}$ \\
\hline 2 & $\begin{array}{l}\text { Slowing down of } \\
\text { organ functions }\end{array}$ & Normal & $\begin{array}{l}\text { Consumption of } \\
\text { internal energy } \\
\text { reserves }\end{array}$ & $\begin{array}{l}\text { With Symptoms in } \\
\text { other organ }\end{array}$ \\
\hline 3 & $\begin{array}{l}\text { Slowing down of } \\
\text { organ functions }\end{array}$ & Normal & $\begin{array}{l}\text { Consumption of } \\
\text { external energy } \\
\text { reserves }\end{array}$ & $\begin{array}{l}\text { With Symptoms in } \\
\text { same organ }\end{array}$ \\
\hline 4 & $\begin{array}{l}\text { Reversible cellular } \\
\text { lesion }\end{array}$ & Little alterations & $\begin{array}{l}\text { Consumption of } \\
\text { blood reserves }\end{array}$ & Curable disease \\
\hline 5 & $\begin{array}{l}\text { Irreversible cellular } \\
\text { lesion }\end{array}$ & $\begin{array}{l}\text { Excessive } \\
\text { alteratioon }\end{array}$ & $\begin{array}{l}\text { Metabolic } \\
\text { exhaustion }\end{array}$ & Incurable disease \\
\hline
\end{tabular}

Table 1. Progression from Health to disease

This anemia or Blood deficiency could be only in the energy level and many times cannot be detected by laboratorial exams, because according to the progression from health to disease, as you can see in the Table 1, the first three phases the patients have symptoms, but the laboratorial exams are normal. When there are alterations on the laboratorial exams, the energies alterations could be presented for five years or more. This means that patient with normal hemoglobin level does not mean that the patient do not have anemia. [3-9]

Another recommendation is to ingest water at temperature of 32 to $36^{\circ} \mathrm{C}$ because the energy of the water is Cold and when ingested at room temperature or colder, can induce the Spleen-pancreas deficiency, leading to deficiency of Blood in the future. The second reason for this change is that the pain in the joints could worse with the ingestion of Cold water, because of entrance of more external Cold pathogenic factor through the water, worsening these symptoms. [12,15]

In most cultures around the world, the temperature of the water is not considered an important factor in the water intake routine. In Brazil, for example, most people drink cold water, usually with ice or in very low temperatures, keeping the water in the refrigerator.
According to traditional Chinese medicine, the quantity of water intake and the water temperature are equally important. The lack of water ingestion may cause Yin deficiency, because the Yin energy is produced in the Kidney, and one of the ways of nourishment to the Kidney is water. The chronic ingestion of cold water is also not beneficial to the body, as the cold water can lead to Spleen-pancreas deficiency. [16]

In relation to Yin and Yang energies that was also reduced in this patient of the case report, these two energies are produced by the Kidney energy meridian or the second chakra. The foods that can induce these energies deficiencies are coffee, soda and mate tea and that is why the author orientated the patient to avoid these foods. [16]

In addition to food, other factors that interfere with the kidney and cause an energy imbalance in it are sleeping late and short of time, excessive sexual activity, parents' energy at conception, overwork, lack of rest, etc. [16]

The author already published some articles about autoimmune diseases, such as Can Autoimmune Hepatitis Be Treated Without the Use of Corticosteroids and Immunosuppressive Drugs? where the author showed through a case report of a female patient who 
How Can You Treat Rheumatoid Arthritis without Using Corticosteroids and Immunosuppressive Medications?

had severe headache in the parietal region. According to TCM literature, this region corresponds to the Liver meridian. Auricular acupuncture with apex ear bloodletting sessions were started, as well as Chinese dietary counselling. Laboratory tests revealed lowprothrombin time and the presence of antinuclear antibody. But after one month of treatment, the exams were repeated and the antinuclear antibody was negative. The conclusion of this study is that autoimmune hepatitis can be treated without the use of corticosteroids and immunosuppressive drugs according to the teachings of TCM, reducing the side effects caused by these drugs. [17]

A fact that generally goes unnoticed by doctors in Western medicine is the ingestion of cow's milk by the patients, which can aggravate the symptoms of autoimmune diseases, in addition to that according to TCM, all joint pain is related to ingestion of cow's milk. [18]

The most important was the change in diet, taking out the milk. [15]

In the book entitled Milk, food or poison? Robert Cohen says that bovine proteins, in addition to being associated with allergies, are also considered a cause of serious illnesses such as cancer, heart disease and osteoporosis. [18] In thisbook, hesaid that osteoporosis is not a problem that should be associated with a lack of calcium intake, but a result of calcium loss. The huge amounts of milk protein result in a $50 \%$ loss of calcium through urine and the calcium contained in vegetables is easier to be absorbed in addition to the fact that vegetable proteins do not result in calcium loss in the same way as animal proteins. [18]

Robert Cohen also quotes the book Stop Osteoarthritis Now where the authors noted that when people who suffer from arthritis and have a low-fat vegetarian diet and add dairy products to their diet, the disease gets worse. [18]

The other tool used by the author to treat this patient reported in this article was auricular acupuncture with apex ear bloodletting. It is a diagnostic and treatment system that normalized the energies imbalances though stimulations points in the ear. The points and the meaning of each point to regulate the internal energy of Yin, Yang, Qi and Blood were well described in the article How Do You Treat Back Pain in Your Practice? Part 2, written by the author and published on February 2018 on Medical Acupuncture Journal. [19,20]

It is important to note that the use of Chinese dietary counseling and acupuncture cannot restore energy of

the chakras' energies centers, so the author used another tool to replenish the chakras' energies centers. $[19,20]$

Other tool used by the author to access information about the energy level of the patient of the five massive internal organs in traditional Chinese medicine (Liver, Heart, Spleen, Lung and Kidney) was the use of radiesthesia procedure. This method consists in the use a crystal pendulum in front of each chakra to decode information about the internal energy of each organ. The crystal or made by wood is placed in front of each chakra and according to the movement of the crystal, the energy level is quantified, which can be stopped if that chakra has no energy, be clockwise and thus evaluated on a scale of one to eight, one being the lowest energy level and eight the highest, but if it is in the counterclockwise sense, there may be some interference and should be better evaluated. [3-9, 19-20]

In the article The Geometry of Emotions: Using Chakra Acupunctureand 5-Phase Theory to Describe Personality Archetypes for Clinical Use, written by Christopher R Chase and published by Medical Acupuncture Journal, the author of this article is correlating the chakras energies centers with the five elements in TCM. Using this correlation, the author begins the measure the energy of each chakra and indirectly, is measuring the energy of each massive organ in TCM, demonstrated in the Figure 8. [21]

The description of this method was well described in the article Why Are Diabetic Patients Still Having Hyperglycemia despite Diet Regulation, Antiglycemic Medication and Insulin? written by the author and published at International Journal of Diabetes \& Metabolic Disorders on March 2018. [9]

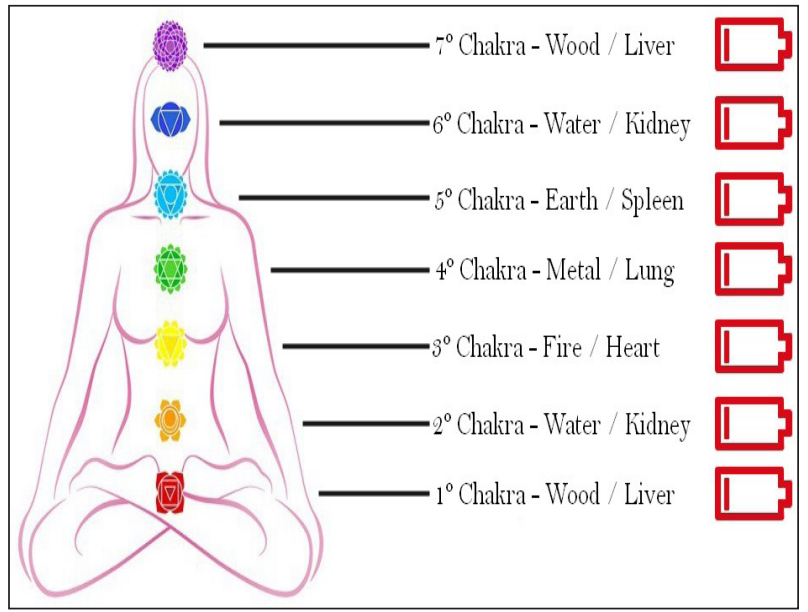

Figure 8. Results of his radiesthesia procedure measurement 
How Can You Treat Rheumatoid Arthritis without Using Corticosteroids and Immunosuppressive Medications?

The treatment to replenish the chakras' energies centers were done using highly diluted medications such as homeopathic medications, according to the theory created and written by the author in the article
Constitutional Homeopathy of the Five Elements based on Traditional Chinese Medicine, published by Acta Scientific Medical Sciences on July 2020. [22] The medications used are described in the table 2.

\begin{tabular}{|c|c|c|c|}
\hline Chakras & Five Elements & Homeopathy Medications & Crystal Based Medications \\
\hline $1^{\circ}$ Chakra & Wood / Liver & Phosphorus & Garnet \\
\hline $2^{\circ}$ Chakra & Water / Kidney & Natrium muriaticum & Orange Calcite \\
$3^{\circ}$ Chakra & Fire / Heart & Sulphur & Rhodochrosite \\
$4^{\circ}$ Chakra & Metal / Lung & Silicea & Emerald \\
\hline $5^{\circ}$ Chakra & Earth / Spleen & Calcarea carbonica & Blue Quartz \\
$6^{\circ}$ Chakra & Water / Kidney & Tone $2^{\circ}$ chakra & Sodalite \\
\hline $7^{\circ}$ Chakra & Wood / Liver & Tone $1^{\circ}$ chakra & Tiger Eye \\
\hline
\end{tabular}

Table 2. Medications used to replenish the energy of the chakras energies centers, homeopathies and crystal based medications

It is important to use homeopathies according to the theory created by the author entitled Constitutional Homeopathy of the Five Elements Based on Traditional Chinese Medicine, published by Acta Scientific Medical Sciences on June 2020 and the medications needs to be given according to the generation cycle of the five elements. For example, if we start with the medicine Silicea to tone the Lung that corresponds to the fourth chakra, then the physician needs to prescribe Natrum muriaticum to tone the second chakra (kidney), which will pass energy to the first chakra (Liver). To treat the lack of energy in the Liver, the physician will prescribe Phosphorus. After, to tone the energy of the third chakra (Heart) it is necessary to use Sulphur, which passes energy to the fifth chakra (Spleen) who needs to be treated with Calcarea carbonica. This element will send energy to the fourth chakra, closing the cycle. [22] The sequence of the use of homeopathic medications should be done according to the results in the radiesthesia procedure of each patient.

These medications are usually used for a period of one year or more and the duration of the use of this medications will be defined in the next radiesthesia procedure, normally repeated by the author after one year of treatment. Normally, she orientates the patient to continue the medications because in her daily practice, she noticed that the energy measurement in the chakras' energies centers still low, despite the clinical improvement that the patient presented after the beginning of the treatment using these kinds of medications. The author does not know yet what could be the influences that is causing the maintenance of the low level of energy in these patients after the beginning of the treatment. One possible influence that she is observing is that the influence of electromagnetic waves in all environment that the human being is living nowadays could be one of the causes but more studies in this field need to be done to conclude the influences of this kind of waves affecting the human body, but in the energy point of view that is normally not studied yet, due to energy is not seeing by the naked eyes and only experts in this field could understand how to study this kind of influence in the energy point of view. [3-9, 19-20]

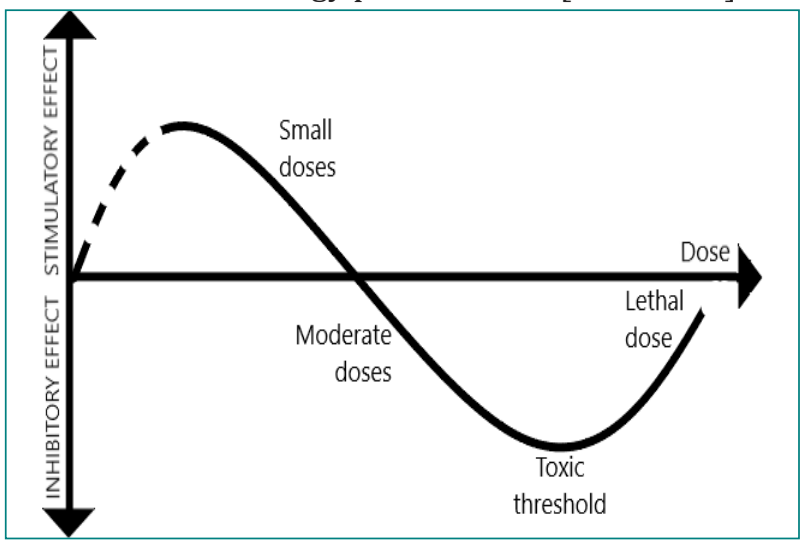

Figure 9. Arndt-Schultz law

The patient reported in this article had knee joint pain, hip in all joints. He was using very strong medications, 
How Can You Treat Rheumatoid Arthritis without Using Corticosteroids and Immunosuppressive Medications?

immunosuppressant and corticosteroids. He told to the author that he had no improvement of his pain condition. In the case of this specific patient, the use of these kind of medications could worsen his case, as they are considered high-concentrated medications, according to the Arndt-Schultz law, showed in the Figure 9. According to this law, created in 1888, by two Chilean researchers, all highly concentrated medications could harm the vital energy of the patient even further, generating more formation of internal Heat, and not improving the symptoms presented by the patient. The use of this kind of drugs themselves were decreasing the patient's energy, as the drugs are metabolized in the Liver and as they are highly concentrated they will consume the patient's energies in addition to forming internal Heat, generating kidney Yin deficiency maintaining the energy imbalance that is causing autoimmune disease. [3-9, 16-17, 19-20, 23]

The patient was recommended to protect even the entire joint with light clothing; for example, avoid shorts that do not cover the knees, blouses with shorter sleeves, use a very light fabric to prevent the Wind from entering. Just by doing these procedures, avoiding milk, raw, Cold things, the invasion of an external pathogen, with less than a month of treatment, it was possible to remove all highly concentrated drugs. In the book The 'Hippocratic' Corpus: Content and Context, written by Elizabeth M. Craik, she mentioned about the influences of the external pathogenic factors in the formation of the diseases. In the book The Yellow Emperor's Classic of Medicine: A New Translation of the Neijing, written Maoshing $\mathrm{Ni}$, the author mentions the influence of the entrance of Wind and Cold inside the body, leading to Bi Syndrome. $[2,10]$

As the patient lives in São Paulo, that is far from the physician $500 \mathrm{Km}$, the treatment was interrupted, but recently talking to the patient's sister who is also the author's patient, it was reported that he is fine and does not need to take any highly concentrated medications (corticosteroids and immunosuppressive medications). She also said that he usually complains pain in the joints only when there is a change in the climate. $[10,14]$

Under normal health conditions the body is not invaded by the six excesses, this happens only when there is an imbalance between the body's $Q i$ and the external pathogenic factor, due to very strong climatic changes, mainly caused by the body's weakness in relation to the factor climatic, in this case the external pathogenic factor penetrates the organism regardless of its intensity. [10,14]

For this reason, the patient should be recommended in addition to the diet and avoid taking Cold, the patient should be suggested to cover the joints so that Wind and Cold do not enter the body and cause Bi syndrome, so the $Q i$ and the Blood to strengthen his immunity so that the Wind and the Cold do not cause so much disease in him, that they are entering due to the deficiency of these two energies according to the TCM. The author when began to treat the chakras' energies deficiencies, is treating the energies deficiencies that the patient has that is propitiating the entrance of the external pathogenic factor (deficiency of Yin, Yang, $Q i$ and Blood) and treating the lack of energies in the chakras' energies centers, the patient will fortify his energy, difficulting the entrance of more external pathogenic factor, that were one of the causes of pain in this patient with rheumatoid arthritis. [10]

Hippocrates owes the first attempt to eliminate supernatural causes under disease, thus attributing a natural cause. Health would result from balances of elements of nature, which, at the time, was contemplated through the combination of four elements - Earth, Water, Fire and Air - outlining their properties: Dry, Humid, Hot and Cold. According to the theorist, the disease was due to the imbalance of the same elements. [2]

The author already publish an article entitled Shoulder and Elbow Tendinitis as Initial Manifestation of Autoimmune Hepatitis published on 2019 by ARC Journal of Orthopedics where the author is showing that tendinitis may be an early manifestation of autoimmune hepatitis. Through a clinical case report of a female patient with chronic pain in shoulder and elbow, resistant to conventional treatments with anti-inflammatories and physiotherapy. She sought acupuncture treatment, and even so, the improvement presented was scarce. In her physical examination, a bulging was detected in the right ear, on the area that corresponds to the Liver, according to auricular medicine. Laboratory tests were requested to see if there could be any undiagnosed liver disease until then. The only exam that was initially altered was the prothrombin time of $89 \%$. More tests were ordered to 
How Can You Treat Rheumatoid Arthritis without Using Corticosteroids and Immunosuppressive Medications?

see whether anti-bodies were present. Anti-nuclear, anti-smooth muscle, anti-microsomal were requested. After the treatment, to the physician's surprise, the patient had a 1:160 anti-nucleus reagent. In this case published in this article, what the author wants to show, is that the Liver has a hollow organ that it commands that is the Gall bladder. This hollow organ is responsible for the tendons, according to TCM reasoning, in the Five Elements theory and that is why disease in the Liver was causing an imbalance in the energy of the Gall bladder, leading the tendinitis symptoms. [7]

The author wants to emphasize that all the tools used in the treatment of this patients such as homeopathy and acupuncture were both recognized by the Brazilian Federal Council since 1980 and 1995, respectively, as medical specialties. [24]

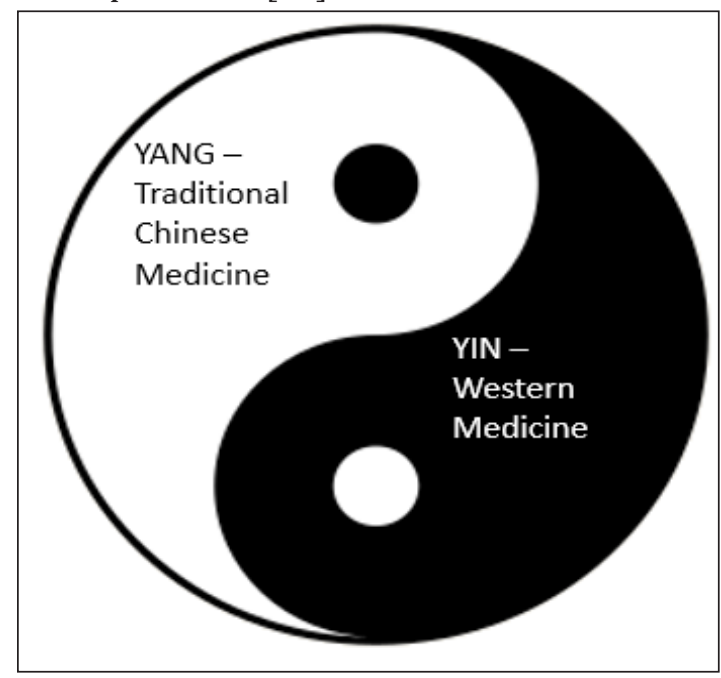

Figure 10. Metaphor of the tree between Western and traditional Chinese medicine

To finalize this article, the author would like to show the metaphor between Yin and Yang theory and Western and traditional Chinese medicines, as you can see in the Figure 10.

In order to treat patients with rheumatoid arthritis in a deeper way, the physician has to comprehend the importance of the different medicine perspectives. The body, is not only physical and constituted by organs and tissues, it is formed by energy. Nowadays, these two perspectives are separated, but they can be complementary. Traditional Chinese medicine can be considered as Yang energy, and Western medicine is considered Yin energy, already materialized. In the same way of the relationship of Yin and Yang, Traditional Chinese medicine starts where Western medicine ends.

\section{ConClusion}

The conclusion of this study is that it is possible to treat rheumatoid arthritis without the use of corticosteroids and immunosuppressive medications. To this end, the physician needs to see the patient as a whole, looking at many other perspectives that normally it is not viewed by the Western medicine such as the kind of diet that the patient is eating, the influence of the external pathogenic factors and the importance to treat the deficiencies of the internal energies of the five massive organs to fortify the energies, mainly $Q i$ and Blood, to prevent the entrance of these external pathogenic factors responsible for the rheumatoid arthritis patients symptoms. The type of diet that the patient should use, needs to be accordingly to their energies disharmonies avoiding all raw foods that could induce more entrance of these external pathogenic factors inside the body. More studies with more patients should be done to evaluate the effectiveness of this kind of treatment in patients with rheumatoid arthritis.

\section{REFERENCES}

[1] Guo Q et al. Rheumatoid arthritis: pathological mechanisms and modern pharmacologic therapies. Bone Res. 2018; 6: 15. Published online 2018 Apr 27.

[2] Craik E. The "Hippocratic" Corpus: Content and Context. Routledge; 2014. 344 p.

[3] Huang WL. The Importance of Correcting Energy Imbalances and Chakras Energy Deficiencies in the Treatment of Patients with Glaucoma. Clin Res Ophthalmol. 2019;2(2):1-9.

[4] Huang WL. The Importance of Correcting Energy Imbalances in the Prevention and Treatment of Câncer. Adv Cancer Res Clin Imaging. 2020;3(1):1-10.

[5] Huang WL. Invasion of Wind and Cold as Cause of Respiratory Tract Infection Outbreak in a School Kinder Garden Group of Kids. Pediatr Res Child Heal. 2020;3(2):1-4.

[6] Huang WL. Chakra's energy deficiency as the main cause of infertility in women. Obstet Gynecol Int J. 2020;11(2):83-91. 
How Can You Treat Rheumatoid Arthritis without Using Corticosteroids and Immunosuppressive Medications?

[7] Huang WL. Can Autoimmune Hepatitis Be Treated Without the Use of Corticosteroids and Immunosuppressive Drugs? Acta Sci Med Sci. 2019;3(7):178-86.

[8] Huang WL. Why patients with knee pain still have symptoms despite the use of anti-inflammatory medications. Int J Orthop Res. 2020;2(2):21-7.

[9] Huang Wei Ling, Why Are Diabetic Patients Still Having Hyperglycemia Despite Diet Regulation, Antiglycemic Medication and Insulin? Int J Diabetes Metab Disord. 2019; 4(2):1-14.

[10] Maoshing NI. The Yellow Emperor's Classic of Medicine: The Essential Text of Chinese Health ahd Healing. Shambala, Boston and London, 1995.

[11] How W, et al. Treating Autoimmune Disease with Chinese Medicine. ELSEVIER. 2011.

[12] Joerg Kastner. Chinese Nutrition Therapy. Dietetics in Traditional Chinese Medicine (TCM). Thieme, 2004.

[13] Zhang E. Bi Syndrome (Arthralgia Syndrome). J Tradit Chin Med. 2010 Jun;30(2):145-52.

[14] Dashtdar M, Dashtdar MR, Dashtdar B, Kardi K, Shirazi MK. The concept of wind in traditional chinese medicine. J Pharmacopuncture. 2016;19(4):293-302.

[15] Perini M. Terapia Dietética Chinesa. Livraria e editora Andreoli. 2020.

[16] Huang WL. Why patients with knee pain still have symptoms despite the use of anti-inflammatory medications. Int J Orthop Res. 2020;2(2):21-7.
[17] Huang WL. Shoulder and Elbow Tendinitis as Initial Manifestation of Autoimmune Hepatitis. ARC J Orthop. 2019;4(2):19-26.

[18] Robert Cohen. Milk, food or poison? Hardcover November 1, 1997

[19] Huang Wei Ling, The Treatment of Asthma Based on Traditional Chinese Medicine and Homeopathy. J Pediat Infants. 2018; 1(1):24-40.

[20] Huang, Wei Ling, How to Treat Low Back Pain in Pregnancy without Using any Anti-Inflammatory Medications? Op Acc J Bio Sci \& Res. 2020; 2(3).

[21] Chase CR. The Geometry of Emotions: Using Chakra Acupuncture and 5-Phase Theory to Describe Personality Archetypes for Clinical Use. Med Acupunct. 2018;30(4):167-78.

[22] Huang WL. Constitutional Homeopathy of the Five Elements based on Traditional Chinese Medicine. Acta Sci Med Sci. 2020;4(7):57-69.

[23] LEESER O. Support of homeopathy by the ArndtSchulz law. National Center for Biotechnology Information. 1953/ Jul 31.

[24] CREMEPE. HOMEOPATIA E ACUPUNTURA RECONHECIDAS E PROCURADAS. November 2006. [Internet]. [Cited 2021 Jan 9]. Avaible from: http://www.cremepe.org.br/2006/11/26/ homeopatia-e-acupuntura-reconhecidas-eprocuradas

Citation: Huang, W. L. How Can You Treat Rheumatoid Arthritis without Using Corticosteroids and Immunosuppressive Medications?. Archives of Anesthesiology. 2020; 3(2): 19-30.

Copyright: (C) 2020: Huang, W. L. This is an open access article distributed under the Creative Commons Attribution License, which permits unrestricted use, distribution, and reproduction in any medium, provided the original work is properly cited. 